\title{
An Air-conditioning Load Forecasting Based on Dynamical Combined Re- sidual Error Correction
}

\author{
Feng Zengxi*, Ren Qingchang and Li Jianwei
}

College of Civil Engineering, Xi'an Univ. of Arch. \& Tech, Xi'an, Shaanxi, 710055, P.R. China

\begin{abstract}
Accurate air-conditioning load forecasting is the precondition for the optimal control and energy saving operation of central air-conditioning system. However, the single forecasting method, such as autoregressive integrated moving average (ARIMA), grey model (GM), multiple linear regression (MLR) and artificial neural network (ANN), has not enough accuracy. In order to improve the accuracy of air-conditioning load forecasting, the combination forecast develops. But so far there are no literatures that explain how to choose the single forecasting methods to build the combination forecast that can further improve the forecasting accuracy. To further improve the forecasting accuracy, a forecasting method with dynamical combined residual error correction is proposed. The residual error correction model and its combination ways are analyzed, and the very high accuracy with mathematical proof is realized in this paper. A case study indicates that the dynamical combination ways proposed in this paper can further improve the accuracy of combination forecasting and satisfy the accuracy requirement of air-conditioning load forecasting.
\end{abstract}

Keywords: Air-conditioning, Combination forecasting, Load, Residual error.

\section{INTRODUCTION}

Because the central air-conditioning system runs under the part load in most cases, the possibility of energy saving can be realized. In order to save the most energy, the airconditioning load must be calculated. Accurate airconditioning load forecasting is the precondition for the optimal control and energy saving operation of central airconditioning system $[1,2]$. Therefore, the air-conditioning load forecasting model must be built to accurately forecast the air-conditioning load before the global optimization control and energy saving of the air-conditioning is done.

Prior research has been done for both the prediction of cooling and heating loads. The approaches discussed in the literature include grey model (GM), multiple linear regression (MLR), autoregressive integrated moving average (ARIMA) and artificial neural network (ANN), etc. These methods have their own scope of application. The grey model was initially used for the heating load forecasting [3-4]. The grey model has two advantages. Firstly, it can be applied in circumstances with relatively little data. Secondly, it can characterize a system with a first-order differential equation. The grey model is usually applied for the long-term forecasting [5-8]. MLR is one simple method that is very easy to be understood and accepted. But it may be a little rough because there are non-linear relationships in practice. MLR is usually applied to forecast the development trend of happenings [9]. ARIMA is an example of statistical forecasting Model [10]. It is very easy and it works with single data.
Because of its rapid forecasting speed, ARIMA is normally used for the short-term prediction.

In recent years, considerable attention has been given to ANNs, unlike energy analysis based on analytical models and industrial methods. Much reported study on ANN applications in the field of thermal load prediction proves that ANN is successful in prediction areas [11-18]. The ANN is good for some tasks while lacking in some others [11]. There are many algorithms for artificial neural network, such as error back propagation (BP), radial basis function (RBF) and Elman. The Elman neural network (ENN) is one simple method to forecast the air-conditioning load [12]. BP is the most popular algorithm applied in load prediction with artificial neural network model [13-15]. However, BP program has some fatal shortcomings such as the difficult determination of good initial weighs and the very slow convergence speed of learning. Aiming at the defects of BP neural network, the radial basis function neural network(RBFNN) has been developed [16-17]. In general, ANN has the advantages of self adaptability and the the ability of learning, but its optimal network structure is difficult to find and it needs a lot of train sample. The accuracy of forecasting can still be improved, and ANN is usually applied for short-term prediction [18].

In order to further improve the accuracy of load forecasting, the combination forecast theory develops. It can improve actually the forecasting accuracy. However, if the viewpoint and method of choosing the single forecasting model that is used to form the combination forecasting method are different, the accuracy of load forecasting is also different. So far there are no literatures that explain how to choose the single forecasting to build the combined forecasting model that can further improve the forecasting accuracy. 


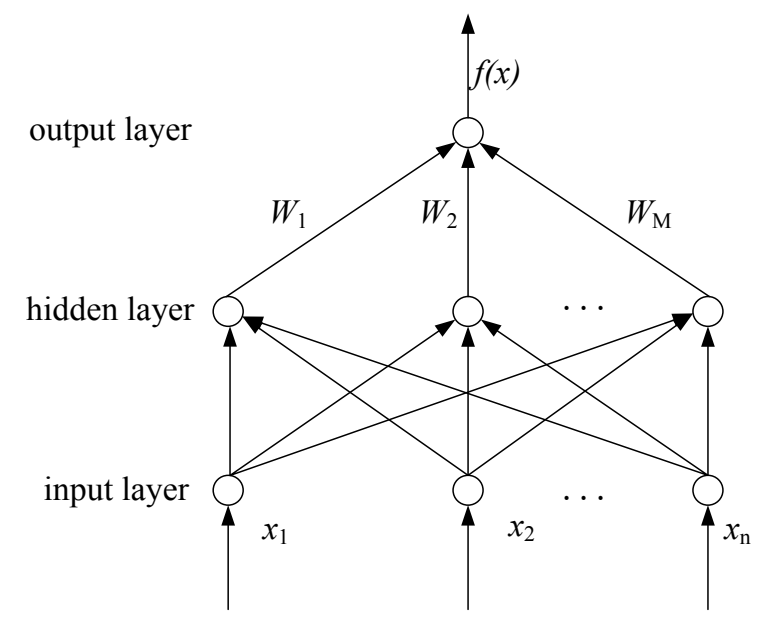

Fig. (1). The structure of RBF network.

The main objective of this paper is to present a new improved method for load forecasting with high precision using the RBFNN with dynamical combined residual error correction. The RBF neural network is chosen to predict the airconditioning load because of its generality and rapid learning rate. At the same time, a combined forecasting model with dynamical combined residual error correction, which is the combination of two models of ARIMA, MLR, Elman and $\mathrm{GM}$, is built to forecast the residual errors of RBF neural network and make the correction. The residual error correction model and its combination ways are analyzed, and the very high accuracy with mathematical proof is realized in this paper. A case study demonstrates that the forecasting accuracy of RBF neural network with dynamical combined residual error correction is better than that of RBFNN with two rigid model correction and RBFNN itself.

\section{RBF NEURAL NETWORK FORECASTING MOD- EL WITH COMBINED RESIDUAL ERROR COR- RECTION}

\subsection{The RBF Neural Network Forecasting Model}

The RBFNN is a kind of feed-forward network. In general, RBF network is a three-layer network. The RBF network structure that is used in this paper [16-19] is shown as Fig. (1).

Here, the input layer consist of $m$ units which represent the elements of the input vector $x$. The hidden layer is composed of $n$ basis functions that execute non-linear mapping. The activation of a hidden neuron is determined by computing the distance between its center vector and the vectors which are yielded by the activation of the input layer. The action of a neuron in the output layer is determined by computing the weighted sum of output of hidden layer. The Gaussian exponential function is often used to define the basis function for the hidden nodes. It is shown as the follow:
$G\left(\| X(t)-T_{i}||\right)=\exp \left[-\frac{1}{2}\left(\frac{\left\|X(t)-T_{i}\right\|}{q}\right)^{2}\right]$

$i=1, \quad 2, \quad \ldots, \mathrm{m}$

where, $T_{i}$ is the center of the $i$ th RBF unit, $T_{i}=\left(t_{1}, t_{2}, \cdots, t_{n}\right)$; $X(t)$ is the input vector of the $t$ th input node, $X(t)=\left(x_{t 1}, x_{t 2}, \cdots, x_{t n}\right) ; q$ is normalization parameter.

The network output $f(x)$ can be built by a linearly weighted sum of the number of basis functions in the hidden layer. The network output values can be computed by Equation (2):

$$
f\left(X(t), w_{i}, T_{i}\right)=\sum_{i=1}^{m} w_{i} G\left(\left\|X(t)-T_{i}\right\|\right)
$$

where, $w_{i}$ is the weight from the $i$ th hidden layer neuron to the output layer neuron, $f(x)$ is the output node in the output layer, $m$ is the node number of hidden layer, $t=1,2, \ldots$, $N, N$ is the total number of samples.

The error is defined as Equation (3)

$$
e(t)=y(t)-f\left(X(t), w_{i}, T_{i}\right)
$$

Where, $y(t)$ is real load, the study process makes the total errors of sample set minimum as Equation (4)

$$
\min E(W)=\frac{1}{2} \sum_{i=1}^{n}|e(t)|^{2}
$$

The RBFNN has two operating modes, named training and testing. The number of RBFs, the output layer weight matrix and the corresponding centers can be determined after the training of RBF network. The details of computing program and algorithm can be found in Refs. [15]. An RBFNN 


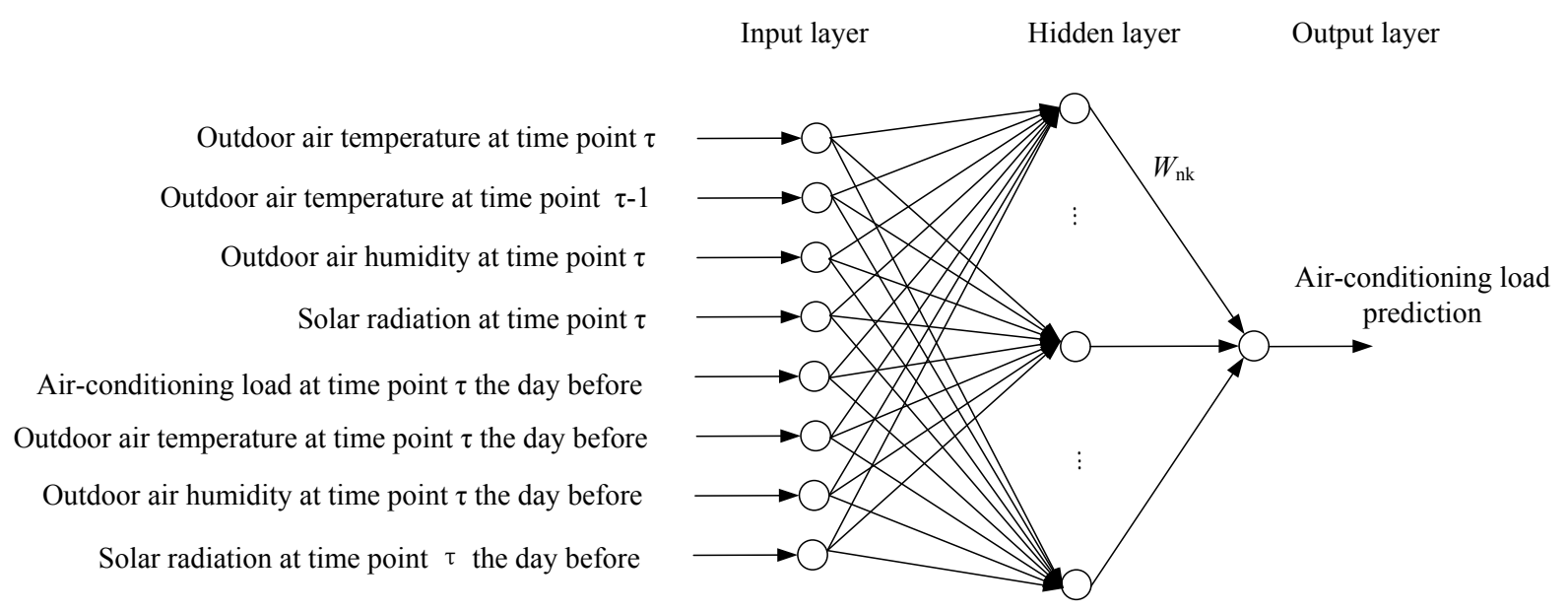

Fig. (2). The RBF model for air-conditioning load forecasting.

can easily calculate the output for any new input vectors after it is determined and well trained.

When the RBFNN is applied to build the air-conditioning load forecasting model, the input layer includes eight neurons that receive input signals of outdoor air temperature at $\tau-1$, outdoor air temperature, outdoor air humidity and solar radiant intensity at $\tau$, outdoor air humidity, outdoor air temperature and solar radiant intensity at $\tau$ the day before, and the air conditioning load at $\tau$ the day before. The output layer has one neuron that calculates the prediction result of airconditioning load. The node number of the hidden layer is calculated by the empirical formula $m=\sqrt{n l}$ where $n$ is the node number of input layer and $l$ is the node number of output layer. The RBF model for air-conditioning load forecasting is shown in Fig. (2).

\subsection{Residual Error Correction with a Combined Model}

Assuming that the forecasted air-conditioning load at the time point $\tau$ is $Q_{R B F}$ and the actual load $Q_{a c t}$, the residual error, $Q_{e}$, can be written as:

$Q_{e}=Q_{R B F}-Q_{a c t}$

Known from Eq. (5), if the residual error, $Q_{e}$, is known, the result, $Q_{a c t}$, at $\tau$ can be written as:

$Q_{a c t}=Q_{R B F}-Q_{e}$

Thus, the residual error, $Q_{e}$, at $\tau$ should be determined by the residual error model, which is also a forecasting problem.

\subsubsection{The Combined Forecasting Model for Residual Error Prediction}

The combination forecasting theory is one very popular subject in the area of forecasting. It is based on a certain linear combination of various results from different forecast models [20-22]. It can greatly improve performance and its mean deviation during a period can minimize because it can take full advantage of the useful information of different models. Its forecasting result is superior to other single forecasting methods.

Now, two typical forecasting methods, including autoregressive integrated moving average (ARIMA) and Elman, are used to establish the residual error model, respectively, at the same time, they are employed to establish the combined forecasting model and make the residual errors correction.

For a certain forecasting problem, assume the residual error in period $t$ is $Q_{e, t}(t=1,2, ., \mathrm{n})$ and the forecasting value of $Q_{e, t}$ in period $t$ by ARIMA and Elman model are $Q_{e, A R I}$ and $Q_{e, E L M}$. Suppose the weights vector is $W=\left[\omega_{A R I}, \omega_{E L M}\right]^{T}$, the combined residual error-forecasting model can be expressed as follows [22]:

$\mathrm{Q}_{\mathrm{e}, \mathrm{t}, \text { combine }}=\omega_{\mathrm{ARI}} \mathrm{Q}_{\mathrm{e}, \mathrm{ARI}}+\omega_{\mathrm{ELM}} \mathrm{Q}_{\mathrm{e}, \mathrm{ELM}}$

where, $Q_{e, A R I} \mathrm{Q}_{\mathrm{e}, \mathrm{ARI}}, Q_{e, E L M}$ are prediction values of residual error forecasted by ARIMA model and Elman model, respectively. $\omega_{A R I}, \omega_{E L M}$ are combination weights of the two forecasting models correspondingly, meanwhile, the constraint equations exist as the following: $\omega_{A R I}+\omega_{E L M}=1.0$, $\omega_{A R I} \geq 0, \omega_{E L M} \geq 0$.

\subsubsection{Determining the Weights in the Combined Model}

In order to dynamically optimize the combination weights according to the historical data, the method of least error square sum (LESS) is used to determine the weights in the combined model [5]. The equivalent equations can be expressed as:

$$
\left\{\begin{array}{l}
\min Z=\sum_{i=1}^{m}\left(Q_{e, t, \text { combine }}(i)-Q_{e, t}(i)\right)^{2} \\
\text { s.t } \\
\omega_{A R I}+\omega_{E L M}=1.0 \\
\omega_{A R I} \geq 0, \omega_{E L M} \geq 0
\end{array}\right.
$$


Supposing that the forecasting deviations of single model for residual error prediction are given as follows:

$\varepsilon_{A R I}=Q_{\mathrm{e}, A R I}-Q_{e, t}$

$\varepsilon_{E L M}=Q_{\mathrm{e}, E L M}-Q_{e, t}$

The deviation of combined residual error forecasting model, $\varepsilon_{\text {combine, }}$ can be got as:

$\varepsilon_{\text {combine }}=Q_{e, t, \text { combie }}-Q_{e, t}$

$=\varepsilon_{A R I} \omega_{A R I}+\varepsilon_{E L M A} \omega_{E L M}$

Thus, Equation (8) can be written in the form of matrix:

$\left\{\begin{array}{l}\min Z=W^{T} H W \\ \text { s.t. } \\ e^{T} W=1.0 \\ W^{T} \geq 0\end{array}\right.$

where, $W=\left[\omega_{A R I}, \omega_{E L M}\right]^{T}, e=[1,1]^{T}$,

$H=\left[\begin{array}{cc}\varepsilon_{A R I}^{2} & \varepsilon_{A R I} \varepsilon_{E L M} \\ \varepsilon_{A R I} \varepsilon_{E L M} & \varepsilon^{2}{ }_{E L M}\end{array}\right]$

The forecasting errors sequence of residual error forecasting model of $Q_{e, A R I}$ and $Q_{e, E L M}$ are defined respectively as Equation (13) and Equation (14).

$\varepsilon_{A R I}=\left(\varepsilon_{A R I, 1}, \varepsilon_{A R I, 2}, \cdots \varepsilon_{A R I, n}\right)$

$\varepsilon_{E L M}=\left(\varepsilon_{E L M, 1}, \varepsilon_{E L M, 2}, \cdots \varepsilon_{E L M, n}\right)$

Because $Q_{e, A R I}$ and $Q_{e, E L M}$ were independent of each other, it is easy to know that the covariance between $Q_{e, A R I}$ and $Q_{e, E L M}$ is equal to zero according to the statistical theory. That is to say, The following Equation (15) can be got.

$\operatorname{Cov}\left(\varepsilon_{E L M}, \varepsilon_{A R I}\right)$

$=E\left\{\left[\varepsilon_{E L E}-E\left(\varepsilon_{E L M}\right)\right]\left[\varepsilon_{A R I}-E\left(\varepsilon_{A R I}\right)\right]\right\}=0$

For stationary series, their deviation series is white noise and the mathematical expectation is equal to zero. Therefore, the following Equation (16) can be got.

$E\left(\varepsilon_{E L M}\right)-E\left(\varepsilon_{A R I}\right)=0$

Combine the Eqs. (15) and (16), the following results can be got:

$$
\begin{aligned}
& E\left(\varepsilon_{E L M} \varepsilon_{A R I}\right)=\operatorname{Cov}\left(\varepsilon_{E L M}, \varepsilon_{A R I}\right) \\
= & \left(\sum_{i=1}^{n} \varepsilon_{E L M, i} \varepsilon_{A R I, i}\right) / n=0
\end{aligned}
$$

Eq.(18) is equal to Equation (17)

$\sum_{i=1}^{\mathrm{n}} \varepsilon_{E L M, i} \varepsilon_{A R I, i}=0$

The Lagrange function is introduced to solve Equation (12) as Equation (19):

$L=W^{T} H W+\lambda\left(e^{T} W-1\right)$ (19),

Then the Equation (20) can be got by solving Equation

$$
\left\{\begin{array}{l}
\frac{\partial L}{\partial W}=2 H W+\lambda e=0 \\
\frac{\partial L}{\partial \lambda}=e^{T} W-1=0
\end{array}\right.
$$

The weights, $\omega_{A R I}, \omega_{E L M}$ in the combined forecasting model can be calculated as:

$W=\left[\varepsilon_{\mathrm{ARI}}, \varepsilon_{E L M}\right]^{T}=\left(H^{-1} e\right) /\left(e^{T} H^{-1} e\right)$

According to Equations (7) and (21), the combined model can be built to revised the air-conditioning forecasting load of RBFNN.

\subsection{The Forecasting Error of RBFNN Forecasting Model with Combined Residual Error Correction}

Supposing that the relative forecasting error of ELMAN is denoted as $\delta E_{E L M}$ and the relative forecasting error of ARIMA is denoted as $\delta E_{A R I}$, the combined relative forecasting error, $\delta E_{z}$, can be expressed as:

$\delta E_{z}=\omega_{\mathrm{ARI}} \delta E_{A R I}+\omega_{E L M} \delta E_{E L M}$

Assuming the forecasted load by RBFNN is denoted as $Q_{R N N, f}$ at time $\tau$ and the actual load is denoted as $Q_{\text {act, }}$, the relative forecasting error of RBFNN, $\delta E_{R N N}$, can be written as:

$\delta E_{R N N}=\frac{\left|\Delta E_{R N N, a c t}\right|}{Q_{a c t}}$

where, $\Delta E_{R N N, \text { act }}$ is the actual residual error of RBFNN at time $\tau$. $\Delta E_{R N N, a c t}=Q_{R N N}-Q_{a c t}$.

When the combined forecasting model is used to predict the actual residual error, $\Delta E_{R N N, \text { act }}$ the relative forecasting error, $\delta E_{\mathrm{Z}}$, can be written as:

$\delta E_{z}=\frac{\left|\Delta E_{z}-\Delta E_{R N N, a c t}\right|}{\left|\Delta E_{R N N, a c t}\right|}$ 
where, $\Delta E_{z}$ is the forecasted residual error of RBFNN by the combined forecasting model.

When $\Delta E_{z}$ is used to correct the forecasted airconditioning load by RBFNN, the ultimate residual error of RBFNN can be expressed as:

$\Delta E_{\mathrm{cor}}=\Delta E_{R N N, \text { act }}-\Delta E_{z}$

Thus, the relative forecasting error of RBFNN with combined-model correction, $\delta E_{R B F N N}^{*}$, can be written as:

$\delta E_{R N N}^{*}=\frac{\left|\Delta E_{R N N, a c t}-\Delta E_{z}\right|}{Q_{a c t}}$

According to Equations (22), (24) and (26), the following equation can be got:

$\delta E_{R N N}^{*}=\frac{\delta E_{\mathrm{z}}\left|\Delta E_{R N N, a c t}\right|}{Q_{a c t}}=\delta E_{z} \delta E_{R N N}$

In accordance with the combination theory, Equation (28) exists:

$\max \left(\delta E_{A R I}, \delta E_{E L M}\right) \geq \delta E_{z} \geq \min \left(\delta E_{A R I}, \delta E_{E L M}\right)$

Assuming the following conditions are given:

$\delta E_{A R I}<1 \& \delta E_{E L M}<1$

Equation (30) can be deduced from Equations (26), (27):

$\delta E_{R N N}^{*}<\delta E_{R N N}$

Equation (30) shows that the forecasting accuracy of RBFNN can be improved after the residual error correction by the combined forecasting model.

\subsection{The Combination Ways and Dynamical Combined Residual Error Correction}

According to the above knowledge, the forecasting accuracy can be improved after the residual error correction by the combined forecasting model. However, as mentioned, each forecasting model has own characteristic and application range. In addition, some influence factors of every single forecasting model are uncertain and random, which leads to the unstable forecasting accuracy. Therefore, if the number of single forecasting models that are used to finish the residual error correction is fixed, how to choose the single forecasting model in multiple forecasting models to form the combined forecasting model? This paper takes the combined forecasting model that is made up of two single forecasting models for example and proves that residual error correction by the dynamic combined forecasting model can further improve the forecasting accuracy.

For the air-conditioning load prediction in certain period, suppose there are four forecasting models, and their forecast- ing relative errors are respectively $\delta E_{1}, \delta E_{2}, \delta E_{3}, \delta E_{4}$. If the following conditions are satisfied:

$\delta E_{1} \leq \delta E_{3}$

$\delta E_{1} \leq \delta E_{4}$

$\delta E_{2} \leq \delta E_{3}$

$\delta E_{2} \leq \delta E_{4}$

Assume

$\omega_{1}+\omega_{2}=1$

where $0 \leq \omega_{1} \leq 1,0 \leq \omega_{2} \leq 1$.

$\omega_{3}+\omega_{4}=1$

where $0 \leq \omega_{3} \leq 1, \quad 0 \leq \omega_{4} \leq 1$.

Known from Equations (31), (32), (33), (34), (35) and (36), the following result can be got:

$\omega_{1} \delta E_{1}+\omega_{2} \delta E_{2} \leq \omega_{3} \delta E_{3}+\omega_{4} \delta E_{4}$

where $\omega_{1}, \omega_{2}, \omega_{3}, \omega_{4}$ are respectively the weights of forecasting model 1 , forecasting model 2 , forecasting model 3 and forecasting model 4.

According to the Equation (22), the following Equations (38) and (39) can be got:

$\delta E_{Z, 12}=\omega_{1} \delta E_{1}+\omega_{2} \delta E_{2}$

$\delta E_{Z, 34}=\omega_{3} \delta E_{3}+\omega_{4} \delta E_{4}$

Combine the Equations (37), (38) and (39), the following condition can be given:

$\delta E_{Z, 12}=\omega_{1} \delta E_{1}+\omega_{2} \delta E_{2} \leq \omega_{3} \delta E_{3}+\omega_{4} \delta E_{4}=\delta E_{Z, 34}$

Where $\delta E_{Z, 12}$ is the combined relative forecasting error based on the forecasting model 1 and forecasting model 2, $\delta E_{Z, 34}$ is the combined relative forecasting error based on the forecasting model 3 and forecasting model 4 .

The following Equations (41) and (42) can be got according to Equation (27)

$$
\begin{aligned}
& \delta E_{R N N, 12}^{*}=\frac{\delta E_{\mathrm{z}, 12}\left|\Delta E_{R N N, a c t}\right|}{Q_{a c t}}=\delta E_{\mathrm{z}, 12} \delta E_{R N N} \\
& \delta E_{R N N, 34}^{*}=\frac{\delta E_{\mathrm{z}, 34}\left|\Delta E_{R N N, a c t}\right|}{Q_{a c t}}=\delta E_{\mathrm{z}, 34} \delta E_{R N N}
\end{aligned}
$$

Combine the Equations (40), (41) and (42), the following results can be got: 


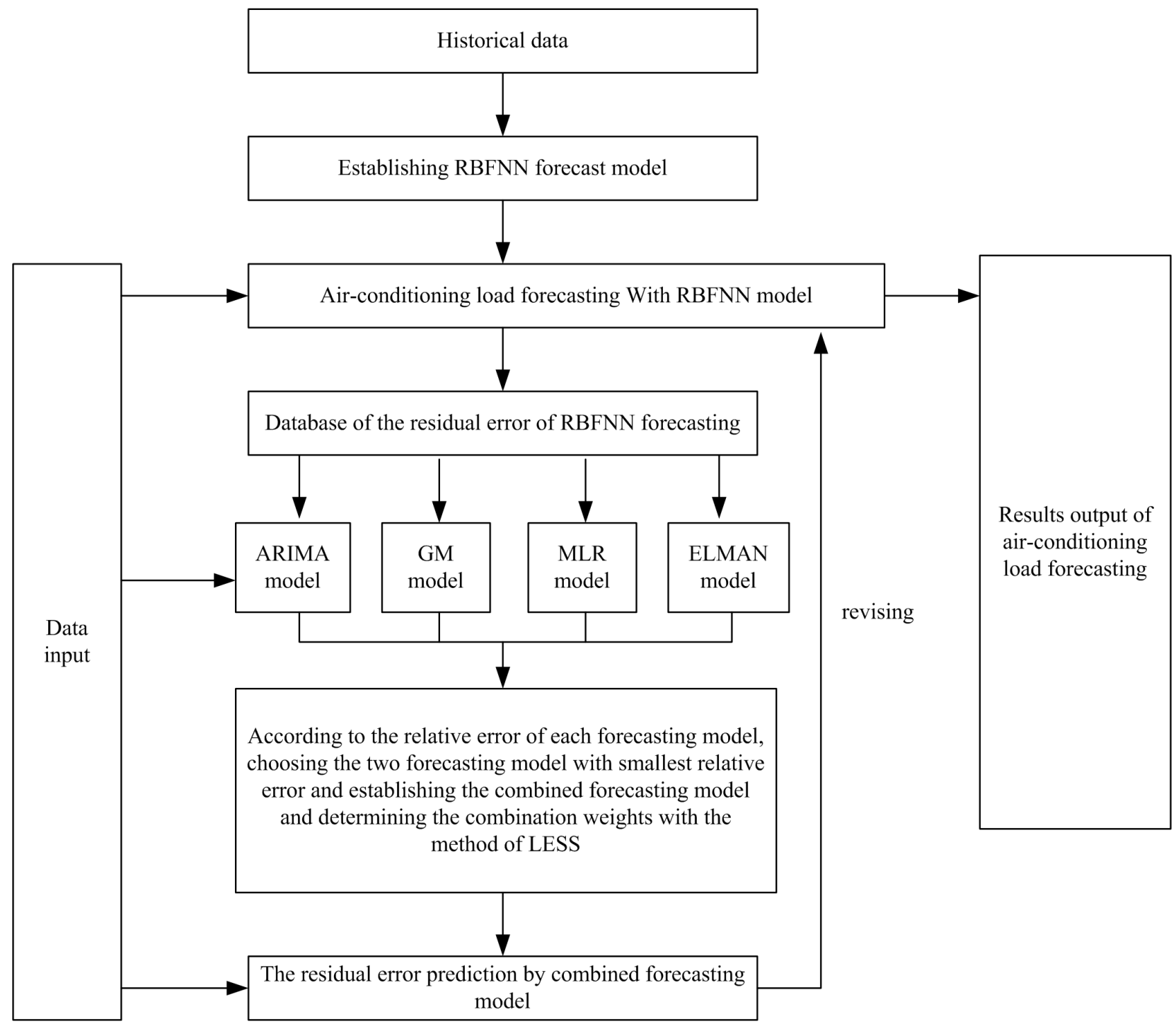

Fig. (3). Forecasting flowchart of RBFNN model with dynamical combined residual error.

$\delta E_{R N N, 12}^{*} \leq \delta E_{R N N, 34}^{*}$

After the residual error correction by different combination, the range of relative forecasting errors $\delta E_{R N N}^{*}$ is given:

$\min \left(\delta E_{1}, \delta E_{2}\right) \delta E_{R N N} \leq \delta E_{R N N, 12}^{*} \leq \max \left(\delta E_{1}, \delta E_{2}\right) \delta E_{R N N}$

$\min \left(\delta E_{3}, \delta E_{4}\right) \delta E_{R N N} \leq \delta E_{R N N, 34}^{*} \leq \max \left(\delta E_{3}, \delta E_{4}\right) \delta E_{R N N}$

Combine Equations (43), (44) and (45), Equation (46) comes into existence:

$\min \left(\delta E_{1}, \delta E_{2}\right) \delta E_{R N N} \leq \delta E_{R N N, 12}^{*} \leq \max \left(\delta E_{1}, \delta E_{2}\right) \delta E_{R N N}$

$\leq \min \left(\delta E_{3}, \delta E_{4}\right) \delta E_{R N N} \leq \delta E_{R N N, 34}^{*} \leq \max \left(\delta E_{3}, \delta E_{4}\right) \delta E_{R N N}$

The above mathematical derivation proves that if the number of single forecasting models that are used to finish the residual error correction is fixed, the single forecasting models whose relative forecasting errors are smaller can be chosen to form the combined forecasting model that can further improve the forecasting accuracy. Therefore, the following idea can be got: the single forecasting models that are used to revise the residual error correction should be chosen dynamically according to respectively relative forecasting errors in different period, so that the combined forecasting accuracy can be further improved.

\section{THE FORECASTING FLOWCHART OF RBFNN MODEL WITH DYNAMICAL COMBINED RESIDU- AL ERROR CORRECTION}

There are two main aspects on the air-conditioning load forecasting with RBFNN model with dynamical combined residual error correction. One is the RBFNN forecasting model, the other is the dynamical combined residual error forecasting model among different period. The forecasting result of RBFNN forecasting model is basis, and it is revised by the combined residual error. The detail forecasting flowchart is showed in Fig. (3). 

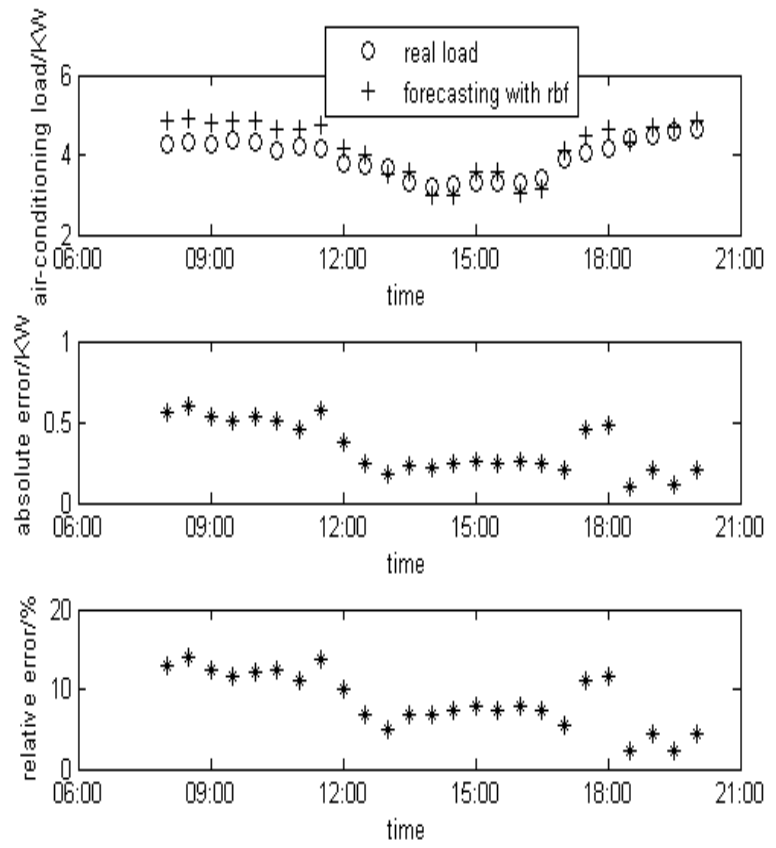

Fig. (4). The result of RBFNN forecasting with no correction.
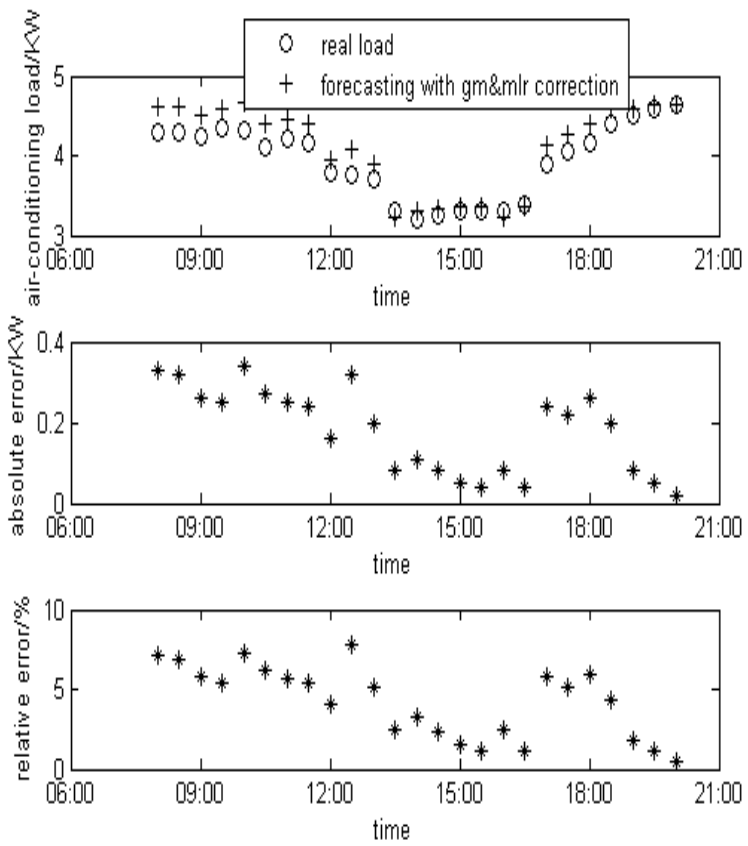

Fig. (5). The result of RBFNN forecasting with GM\&MLR correction.

\section{A CASE STUDY}

The load forecasting method is applied in one central airconditioning experiment platform. The forecasting result of RBFNN forecasting model is basis, and the results of other combined forecasting models make the corrections for RBFNN forecasting. In order to analyze the result of different combined residual error corrections, there are three combined ways to revise the forecasting result of RBFNN forecasting model. The first combined residual error is finished with ARIMA and ELMAN model. The second combined residual error is finished with MLR and GM model. The third combined residual error is finished with dynamical combination that is presented in this paper. It chooses dynamically two single forecasting models with less average relative errors in four forecasting models that are used in this paper during different prediction period.

Three-year datum was used to train the RBFNN forecasting model and other forecasting models that are used in this paper. They were used to forecast ten-hour air conditioning of one day in the winter. Fig. (4) shows the air-conditioning load forecasting results of RBFNN model. The forecasting accuracy of RBFNN model is not enough high. Almost one half of relative errors are above $10 \%$. Figs. (5) depicts the forecasting results of RBFNN model after combined correc- 

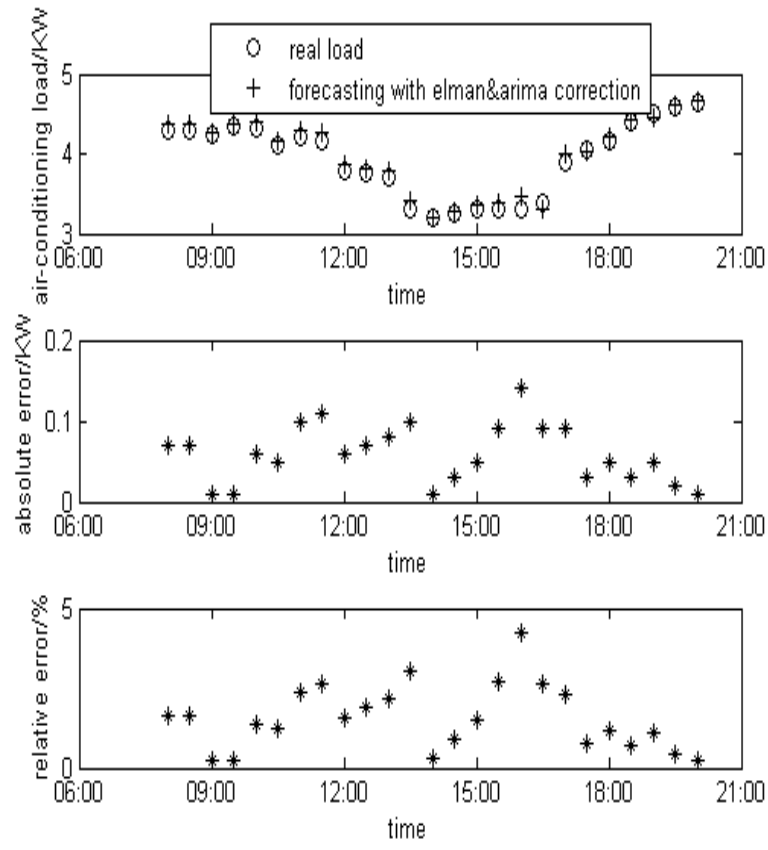

Fig. (6). The result of RBFNN forecasting with ELMAN\&ARIMA correction.
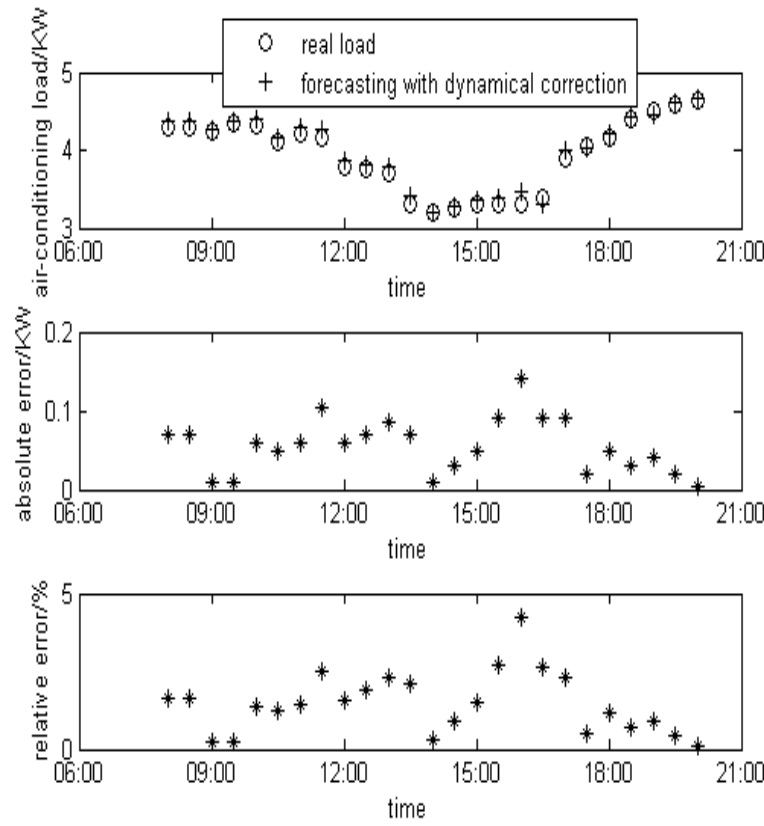

Fig. (7). The result of RBFNN forecasting with dynamical correction

tion of MLR and GM. Figs. (6) depicts the forecasting results of RBFNN model after combined correction of ARIMA and ELMAN. Figs. (5-6) show that the forecasting accuracy of RBFNN is much lower than that of RBFNN with combined correction and show that the forecasting accuracy of RBFNN with combined correction of ARIMA and ELMAN is higher than that of RBFNN with combined correction of MLR and GM. Fig. (7) depicts the forecasting results of RBFNN model with the dynamical combined correction. Comparing the absolute errors and relative errors in Figs. (46) with those in Fig. (7), it is easy to get the conclusion that the forecasting accuracy of RBFNN with dynamical combined correction is higher that of RBFNN with fixed correction. Almost all the relative error of RBFNN forecasting with dynamical combined correction can be controlled within $3 \%$.

\section{CONCLUSION}

An air-conditioning load forecasting method with dynamical combined residual correction is proposed in this paper. It uses RBFNN for the forecasting and it uses the dynamical combination for residual correction. The dynamical 
combination consists of two models with least relative errors of ARIMA, ELMAN, MLR and GM for residual in certain period. One case study indicates the forecasting accuracy with dynamical combined residual correction is higher than other forecasting method and almost all the relative errors are controlled within 3\%.

\section{CONFLICT OF INTEREST}

The author confirms that this article content has no conflict of interest.

\section{ACKNOWLEDGEMENTS}

This work is supported by the technology projects of Ministry of construction (2012-K1-35) and the technology projects of Xian city construction (sjw2014028).

\section{REFERENCES}

[1] Y. Iwasaki, S. Kobayashi, and A. Nagaiwa, "Development of district heating and cooling plant operation support system", ASHRAE Trans, vol. 104, no. 1, pp. 5-12, 1998.

[2] T. Y. Chen, and A.K. Athientis, "Ambient temperature and solar radiation prediction for predictive control of HVAC systems and a methodology for optimal building heating dynamic operation", ASHRAE Transactions, vol. 102, no. 2, pp. 5-12. 1996.

[3] J. L. Deng, "Introduction to grey system theory", Journal of Grey System, vol. 1, pp.1-24, 1989.

[4] W. Siqing, W. Xiamin, L. Yuandan, and Y. Changzi, "Grey interval forecasting of loads of building hydrothermal system", System Engineering, vol. 17, no. 3, pp. 60-65, 1999.

[5] Y. Yao, and Z. W. Lian. "An innovative air-conditioning load forecasting model based on RBF neural network and combined residual error correction", International Journal of Refrigeration, vol. 29, pp. 528-538, 2006

[6] F. M. Tseng, H. C. Yu, and G. H. Tzeng, "Applied hybrid grey model to forecast seasonal time series", Technological Forecasting and Social Change, vol. 67, no. 3, pp. 291-302, 2001.

[7] C. T. Lin, and S. Y. Yang, "Forecast of the output value of Taiwan'S opto-electronics industry using the grey forecasting model", Technological Forecasting and Social Change, vol. 70. no. 2, pp. 177-186, 2003.
[8] L. C. Hsu, "Applying the grey prediction model to the global integrated circuit industry", Technological Forecasting and Social Change, vol. 70, no. 6, pp. 563-574, 2003.

[9] J. R. Forrester, and W. J. Wepfer, "Formulation of a load prediction algorithm for a large commercial Building", ASHRAE Transactions, vol. 90, no. 2B, pp. 536-547, 1984.

[10] A. Kimbara, S. Kurosu, and R. Endo, "On-line prediction for load profile of an air-conditioning system", ASHRAE Transactions, vol. 101, no. 2, pp. 198-207, 1995.

[11] M. Kawashima, C. E. Dorgan, and J. W. Mitchell, "Hourly thermal load prediction for the next 24 hours by ARIMA, EWMA, LR, and an artificial neural network", ASHRAE Transactions, vol. 101, no. 1, pp. 187-200, 1995.

[12] X. W. Tang, "Some new results of combination forecasting study", Prediction, vol. 11, no. 5, pp. 39-46, 1992.

[13] M. B. O. Ohlsson, C. O. Peterson, and P. Hong, "Predicting system loads with artificial neural networks-methods and results from 'the great energy predictor shootout", ASHRAE Transactions, vol. 100, pp. 1063-1074, 1994.

[14] M. Kawashima, "Artificial neural network back-propagation model with three--phase annealing developed for the building energy predictor shootout", ASHRAE Transactions, vol. 100, no. 2, pp. 1096$1118,1994$.

[15] T. Saksornchai, W. J. Lee, K. Methaprayoon, J. R. Liao, and R. J. Ross, "Improve the unit commitment scheduling by using the neural-network-based short-term load forecasting", IEEE Transactions on Industrial Applications, vol. 41, no. 1, pp. 169-179, 2005.

[16] M. Bianchini, P. Frasconi, and M. Gori, "Learning without local minima in radial basis function networks", IEEE Transactions on Neural Networks, vol. 6, no. 3, pp. 749-756, 1995.

[17] S. Haykin, Neural networks: a comprehensive foundation. 2nd ed, Prentice Hall, New Jersey, 1999.

[18] C. E. Chiu, D. F. Cook, J. L. Kao, and Y. C. Chou, "Combing a neural network and a rule - based expert system for short-term load forecasting", Computers \& Industrial Engineering, vol. 32, no. 4, pp. 787-797, 1997.

[19] H. Dernuth, and M. Beale, Matlab neural network toolbox, The Math Works Inc., Natick, MA, 1992.

[20] S. Gupia, and P. Wilton, "Combination of forecasts: an extension", Management Science, vol. 33, no. 3, pp. 356-372, 1987.

[21] G. J. Chert, K. K. Li, and T. S. Chung, "Application of all innovative combined forecasting method in power system load forecasting", Electric Power Systems Research, vol. 59, no. 2, pp.131-137, 2001.

[22] J. M. Bates, and C. Granger, "Combination of Forecast", Operation Research Quarterly, vol. 20, pp. 451-468, 1969. 